\title{
ELETROMIOGRAFIA DE SUPERFÍCIE EM PACIENTES PORTADORES DE PARALISIA FACIAL PERIFÉRICA
}

\section{Surface electromyography in peripheral facial paralysis patients}

\author{
Daniele Fontes Ferreira Bernardes ${ }^{(1)}$, Maria Valéria Schmidt Goffi Gomez ${ }^{(2)}$, Ricardo Ferreira Bento ${ }^{(3)}$
}

\begin{abstract}
RESUMO
Objetivo: estudar a atividade eletromiográfica dos músculos frontal, orbicular dos olhos, zigomáticos, orbicular da boca em indivíduos normais e pacientes portadores de paralisia facial e o índice de simetria entre os dois lados da face. Métodos: foram avaliados por meio da eletromiografia de superfície, seis indivíduos sem histórico de alteração na musculatura facial e seis pacientes com paralisia facial periférica. Para a avaliação eletromiográfica foram solicitados os seguintes movimentos (ao esforço máximo): elevação da testa, fechamento de olhos, protrusão labial e retração labial. Resultados: encontrou-se que em indivíduos normais a média dos potenciais eletromiográficos para ambos os lados da face é semelhante, demonstrando que a integridade do nervo facial é fundamental para o equilíbrio da mímica facial. Nos pacientes com paralisia facial a média dos potenciais eletromiográficos para ambos os lados da face é significativamente diferente (evidenciando a falta de inervação neural). Conclusão: os resultados eletromiográficos mostraram diferença estatisticamente significante entres os dois lados da face nos indivíduos normais e nos pacientes com paralisia facial.
\end{abstract}

DESCRITORES: Paralisia Facial; Eletromiografia; Nervo Facial

\section{INTRODUÇÃO}

A falta de movimentos e de expressão de um dos lados da face, assim como as alterações no modo de falar, e, sobretudo, a impossibilidade de usar a mímica facial, constituem desde os primórdios da humanidade, um dos desfiguramentos mais flagrantes. A face revela o íntimo de nossa expressão e é parte essencial da comunicação humana ${ }^{1}$. A expressão facial é o principal meio de comunicação não verbal, representando a forma como os indivíduos são apresentados ao mundo e como são distinguidas as suas emoções ${ }^{2}$.

(1) Fonoaudióloga; Clínica Daniele Bernardes, São Paulo, SP; Mestre em Ciências pela Faculdade de Medicina da Universidade de São Paulo.

(2) Fonoaudióloga do Setor de Audiologia da Divisão de Clínica Otorrinolaringológica do Hospital das Clínicas da Faculdade de Medicina da Universidade de São Paulo, HCFMUSP, São Paulo, SP; Doutora em Ciências pela Universidade Federal de São Paulo.

(3) Médico; Professor Titular da Disciplina de Otorrinolaringologia da Faculdade de Medicina da Universidade de São Paulo, FMUSP, São Paulo, SP; Doutor em Medicina pela Faculdade de Medicina da Universidade de São Paulo.

Conflito de interesses: inexistente
$\mathrm{Na}$ área da fonoaudiologia vários trabalhos abordam a terapia fonoaudilógica como recurso para tratar os pacientes acometidos pela Paralisia Facial Periférica (PFP). A importância do trabaIho miofuncional nos pacientes com PFP antes da regeneração neural, ou seja, na fase flácida, auxilia de maneira significativa a recuperação funcional final ${ }^{3,4}$. O trabalho fonoaudiológico nas paralisias faciais já é consagrado, porém ainda encontram-se dificuldades em obter um método de avaliação que seja prático, objetivo, de baixo custo e que possa ser usado por fonoaudiólogos, no acompanhamento e na determinação da alta.

Vários centros de reabilitação do nervo facial empregam métodos subjetivos de avaliação da PFP, tais como os critérios de classificação de House e Brackmann (HB) ${ }^{5}$ e o Sistema de Graduação Facial de Ross, Fradet e Nedzelski ${ }^{6}$. A classificação de HB e Ross foi utilizada para avaliar o perfil funcional nos indivíduos com paralisia facial tratados em uma abordagem miofuncional. Estes métodos são simples e de baixo custo, porém dependem do julgamento subjetivo do examinador ${ }^{7}$.

A eletromiografia com eletrodos de agulha, os quais são inseridos na pele do paciente, é um exame capaz de demonstrar potenciais de 
fibrilação nos músculos ${ }^{8}$, porém é um exame que não faz parte da prática fonoaudiológica, por ser um exame invasivo. A utilização da eletromiografia de superfície (EMGs) é recente em fonoaudiologia, tendo como objetivo auxiliar no diagnóstico e tratamento dos distúrbios motores orais, nas alterações da deglutição, mastigação e fala ${ }^{9-13}$. Como é um exame indolor e não invasivo, uma vez que o eletrodo de superfície é posicionado sobre a pele permitindo que o paciente execute o movimento ou função solicitada de maneira relaxada, podendo realizá-la repetidas vezes, sendo de grande valia nas avaliações miofuncionais orofaciais ${ }^{14}$. Na face humana a EMGs tem sido muito explorada para fins de reabilitação por meio do biofeedback eletromiográfico ${ }^{15-18}$.

O objetivo deste estudo foi avaliar os valores da atividade eletromiográfica dos músculos frontal, orbicular dos olhos, orbicular da boca em indivíduos normais e pacientes portadores de paralisia facial periférica.

\section{MÉTODOS}

Foi realizado um estudo prospectivo de corte transversal.

Os grupos constituíram-se de seis sujeitos voluntários sadios, sem paralisia facial nem passado de alterações miofuncionais orofaciais (grupo 1-G1) e seis pacientes portadores de PFP (HBV, VI) (grupo 2- G2), encaminhados pelo Ambulatório de Paralisia Facial ao setor de Fonoaudiologia da Divisão de Clínica Otorrinolaringológica do Hospital das Clínicas da Faculdade de Medicina da Universidade de São Paulo.

Critérios de inclusão:

- Paralisia facial periférica unilateral de qualquer etiologia;

- Classificação de HB: V e VI;

- Tempo máximo de evolução da doença em 30 dias.

- Critérios de exclusão:

- Paralisias faciais prévias;

- Paralisia facial periférica congênita;

- Presença de barba;

- Lesões faciais que interfiram no contato dos eletrodos.

A avaliação da atividade elétrica foi realizada por meio de registro eletromiográfico, usando equipamento Miotec com software Miotool $400^{\circledR}$ - de quatro canais com eletrodos de superfície Double Ag/ $\mathrm{AgCl}$, pré-geleificado.

Após a limpeza da pele com álcool a $70^{\circ}$, os eletrodos de superfície foram aderidos em ambos os lados da face, na região dos músculos frontal, orbi-
A pesuisa foi apresentada e aprovada pela Comissão de Ética em Pesquisa sob o protocolo $\mathrm{n} \cong 313 / 05$. Todos os pacientes assinaram o termo de consentimento antes de serem avaliados.

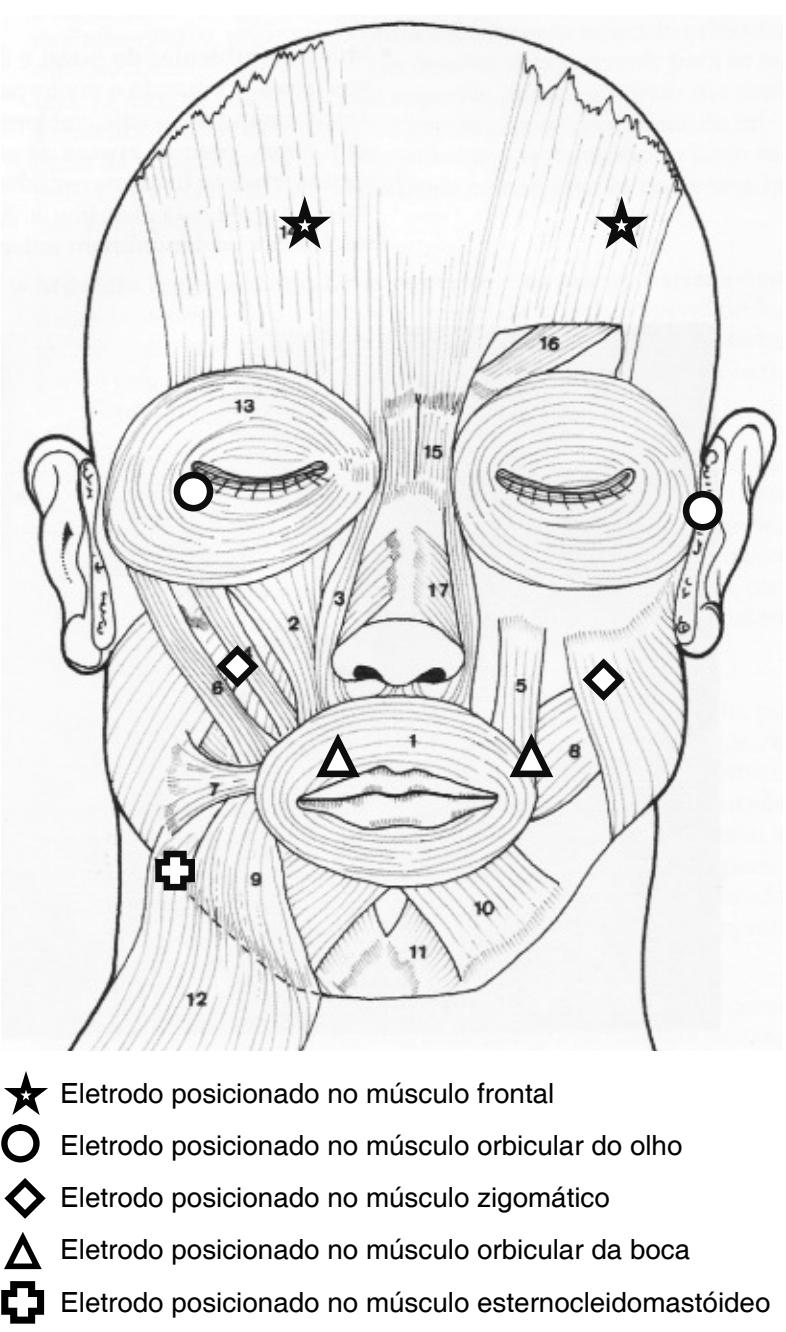

Figura 1 - Posicionamento dos eletrodos nos músculos da face

cular do olho e orbicular da boca simultaneamente, zigomáticos e orbicular dos olhos simultaneamente, o eletrodo terra foi aderido sobre o músculo esternocleidomastóideo (Figura 1).

Os indivíduos executaram sete tarefas ( $\mathrm{T} 1-\mathrm{T} 7)$, ao esforço máximo, durante 8 segundos:

- T1: elevação da testa,

- T2: fechamento forçado dos olhos,

- T3: atividade dos lábios durante fechamento forçado dos olhos

- T4: protrusão labial

- T5: atividade dos olhos durante protrusão labial

- T6: retração labial

- T7: atividade dos olhos durante retração labial 
As respostas eletromiográficas de cada prova de ambos os lados da face expressas em microvolts $(\mu \mathrm{V})$ resultantes da média da atividade elétrica durante 08 segundos foram submetidas à análise estatística realizada com o teste T- Student, considerando-se $p<0,05$ estatisticamente significante.

Foram analisados os dados da atividade eletromiográfica em root mean square (RMS), e calculado o índice de simetria entre os lados. No G1 foi utilizada a razão dos valores da atividade eletromiográfica $(\mathrm{em} \mu \mathrm{v})$ dos movimentos lado direito (D) e lado esquerdo (E), e para o G2 foi usada a razão dos valores da atividade eletromiográfica $(\mu \mathrm{v})$ dos movimentos, lado da paralisia $(P)$ sobre a atividade eletromiográfica do lado normal $(\mathrm{N})$. A partir da razão $D / E$ e da razão $P / N$ foram calculados os índices de simetria para cada grupo.

\section{RESULTADOS}

A idade da população estudada variou entre 36 e 67 anos no G1 e entre 33 a 58 no G2. Com relação ao sexo, no $\mathrm{G} 1$, quatro eram do sexo feminino e dois eram do sexo masculino, no G2 três eram do sexo feminino e três eram do sexo masculino.

A Tabela 1 mostra o índice de simetria D/E no grupo 1 e $\mathrm{P} / \mathrm{N}$ no grupo 2 dos valores médios da atividade eletromiográfica em todos os músculos avaliados. A diferença entre os grupos foi estatisticamente significante $(p<0,05)$.
A Tabela 2 mostra as médias das atividades eletromiográficas em valores absolutos ( $\mu v)$ no grupo G2 foi menor que no G1 em ambos os lados da face em todos os movimentos testados, exceto no sorriso.

\section{DISCUSSÃO}

O acometimento do sétimo nervo resulta em perda da informação neural para os 23 músculos faciais inervados por ele, sendo que toda a hemi face ipsilateral à lesão estará comprometida, em maior ou menor grau de comprometimento ${ }^{19}$. A avaliação clínica desse comprometimento é subjetiva, variando de examinador para examinador e nos diferentes momentos da evolução da doença. A determinação da evolução e quantificação da perda funcional exigem métodos de avaliação que tragam evidências da condição clínica ${ }^{20}$.

A análise eletromiográfica de superfície veio preencher esta lacuna, pois pode ser realizada em todos os músculos esqueléticos, evidenciando pequenas assimetrias faciais em todos os segmentos desejados, não só em pacientes portadores de PFP, mas também em indivíduos normais 12,20-22.

Outra vantagem da EMGs é ser um método objetivo, no qual é possível visualizar a atividade muscular de ambos os lados da face, compará-la e quantificar a sua recuperação, importante tanto para o paciente quanto para o profissional.

\section{Tabela 1 - Índice de simetria (D/E no grupo 1 e P/N no grupo 2) dos valores médios da atividade eletromiográfica em todos os músculos avaliados no G1 e no G2}

\begin{tabular}{|c|c|c|c|}
\hline & G1 & G2 & p \\
\hline Elevação de testa & $0,88^{ \pm} 0,29$ & $0,28^{ \pm} 0,11$ & 0,001 \\
\hline Olhos forçados & $0,83^{ \pm} 0,23$ & $0,32^{ \pm} 0,25$ & 0,005 \\
\hline $\begin{array}{l}\text { Atividade dos lábios } \\
\text { durante o fechamento } \\
\text { dos olhos }\end{array}$ & $0,97^{ \pm} 0,27$ & $0,63^{ \pm} 0,28$ & 0,055 \\
\hline Protrusão de lábios & $0,97^{ \pm} 0,15$ & $0,32^{ \pm} 0,10$ & $<0,001$ \\
\hline $\begin{array}{c}\text { Atividade dos olhos } \\
\text { durante a protrusão } \\
\text { labial }\end{array}$ & $1,17^{ \pm} 0,65$ & $0,86^{ \pm} 0,47$ & 0,371 \\
\hline Retração labial & $0,93^{ \pm} 0,20$ & $0,22^{ \pm} 0,09$ & $<0,001$ \\
\hline $\begin{array}{l}\text { Atividade dos olhos } \\
\text { durante a retração labial }\end{array}$ & $1,11^{ \pm} 0,28$ & $0,45^{ \pm} 0,14$ & 0,001 \\
\hline $\begin{array}{l}\text { G1: indivíduos voluntários sadios } \\
\text { G2: pacientes portadores de para } \\
\text { D: lado direito da face } \\
\text { E: lado esquerdo da face } \\
\text { : Iado paralisado da face } \\
\text { v: lado normal da face } \\
0<0,05 \\
\text { este 't'de Student }\end{array}$ & periférica & & \\
\hline
\end{tabular}


Tabela 2 - Médias das atividades eletromiográficas em valores absolutos em ( $\mu \mathrm{v})$ (e desvios-padrão) em ambos os grupos em ambos os lados da face em todos os movimentos testados

\begin{tabular}{|c|c|c|c|c|}
\hline & \multicolumn{2}{|c|}{ G1 } & \multicolumn{2}{|c|}{ G2 } \\
\hline & $\begin{array}{c}D \\
\text { Média ( } \pm \text { DP) }\end{array}$ & $\begin{array}{c}E \\
\text { Média ( } \pm \text { DP) } \\
\end{array}$ & $\begin{array}{c}P \\
\text { Média ( } \pm \text { DP) } \\
\end{array}$ & $\begin{array}{c}\mathrm{N} \\
\text { Média ( } \pm \mathrm{DP}) \\
\end{array}$ \\
\hline Elevação de testa & $\begin{array}{c}147,48 \\
( \pm 102,92)\end{array}$ & $\begin{array}{c}135,87 \\
( \pm 107,99)\end{array}$ & $\begin{array}{c}65,98 \\
( \pm 87,81)\end{array}$ & $\begin{array}{c}113,91 \\
( \pm 38,31)\end{array}$ \\
\hline Olhos forçados & $\begin{array}{c}158,01 \\
( \pm 1,95)\end{array}$ & $\begin{array}{c}126,52 \\
( \pm 34,08)\end{array}$ & $\begin{array}{c}72,34 \\
( \pm 74,14)\end{array}$ & $\begin{array}{c}112,55 \\
( \pm 109,65)\end{array}$ \\
\hline $\begin{array}{l}\text { Atividade dos } \\
\text { lábios durante o } \\
\text { fechamento dos } \\
\text { olhos }\end{array}$ & $\begin{array}{c}50,50 \\
( \pm 58,01)\end{array}$ & $\begin{array}{c}46,79 \\
( \pm 40,52)\end{array}$ & $\begin{array}{c}23,42 \\
( \pm 2,85)\end{array}$ & $\begin{array}{c}37,44 \\
( \pm 24,02)\end{array}$ \\
\hline Protrusão labial & $\begin{array}{c}227,55 \\
( \pm 31,60)\end{array}$ & $\begin{array}{l}213,05 \\
( \pm 8,42)\end{array}$ & $\begin{array}{c}135,63 \\
( \pm 71,75)\end{array}$ & $\begin{array}{c}160,17 \\
( \pm 11,68)\end{array}$ \\
\hline $\begin{array}{l}\text { Atividade dos } \\
\text { olhos durante a } \\
\text { protrusão labial }\end{array}$ & $\begin{array}{c}36,76 \\
( \pm 2,48)\end{array}$ & $\begin{array}{c}39,51 \\
( \pm 37,63)\end{array}$ & $\begin{array}{c}32,55 \\
( \pm 4,48)\end{array}$ & $\begin{array}{c}43,46 \\
( \pm 3,81)\end{array}$ \\
\hline Retração labial & $\begin{array}{c}150,64 \\
( \pm 27,11)\end{array}$ & $\begin{array}{l}142,56 \\
( \pm 6,38)\end{array}$ & $\begin{array}{c}87,61 \\
( \pm 39,22)\end{array}$ & $\begin{array}{c}169,77 \\
( \pm 86,60)\end{array}$ \\
\hline $\begin{array}{l}\text { Atividade dos } \\
\text { olhos durante a } \\
\text { retração labial }\end{array}$ & $\begin{array}{c}72,14 \\
( \pm 8,29)\end{array}$ & $\begin{array}{c}78,56 \\
(+14,21)\end{array}$ & $\begin{array}{c}55,64 \\
( \pm 66,19)\end{array}$ & $\begin{array}{c}66,38 \\
( \pm 29,08)\end{array}$ \\
\hline
\end{tabular}

G1: indivíduos voluntários sadios

G2: pacientes portadores de paralisia facial periférica

D: lado direito da face

E: lado esquerdo da face

$\mathrm{P}$ : lado paralisado da face

$\mathrm{N}$ : lado normal da face

$\mu \mathrm{V}$ : microvolts

Neste estudo observou-se que nos indivíduos normais a média eletromiográfica para ambos os lados da face são semelhantes, demonstrando que a integridade do nervo facial é fundamental para o equilíbrio da mímica facial ${ }^{23}$.

Nos pacientes com PFP na fase flácida, a média eletromiográfica para ambos os lados da face são significativamente diferentes, como era esperado, demonstrando a lesão do nervo facial e o comprometimento da musculatura facial ${ }^{24}$.

O índice simetria facial $D / E$ nos indivíduos do $G 1$ (sem PFP) gira em torno dos 0,83 a 0,97 , enquanto nos indivíduos do G2 (com PFP) o índice de simetria entre $\mathrm{P} / \mathrm{N}$ gira em torno de 0,28 a 0,32 .

A face humana normal tem $6 \%$ de assimetria entre os lados, esta diferença se dá pela assimetria anatômica, contração muscular assimétrica causada pela força não balanceada e por diferenças técnicas nos procedimentos. A comparação lado a lado de uma mesma face é mais consistente do que a comparação da mesma distância em faces diferentes ${ }^{24}$.
A EMGs foi realizada com o objetivo de examinar se houve melhora na atividade muscular dos pacientes após um procedimento de cirurgia estética. Os resultados demonstraram aumento da atividade elétrica do músculo zigomático maior em todos os pacientes, tanto no pico máximo quanto nas médias eletromiográficas ${ }^{25}$.

Durante a execução das provas T1 a T7 a atividade eletromiográfica dos músculos da hemiface normal dos pacientes com PFP foi menor na maioria dos movimentos, exceto na função do sorriso. Esse achado é importante suporte na reabilitação da PFP, pois se deve orientar aos pacientes a diminuírem a ação dos zigomáticos durante o sorriso, promovendo assim uma menor assimetria facial em ambos os lados.

O Biofeedback EMG foi indicado para o tratamento da paralisia facial com resultados bastante positivos ${ }^{17,18}$. O uso da técnica do Biofeedback EMG associado à Fonoaudiologia e Estética da Face com o objetivo de restabelecer a simetria do sorriso em um paciente com sequela de paralisia 
facial periférica demonstrou ser eficaz para o tratamento das assimetrias faciais ${ }^{18}$.

A maior importância atribuída à eletromiografia de superfície na avaliação é a possibilidade de discutir a alta de forma objetiva e concreta com o paciente. Quando a atividade eletromiográfica da hemiface paralisada aumentar e se aproximar do valor da hemiface oposta, é um sinal que está se alcançando a simetria facial e os exercícios miofuncionais devem ser diminuídos até a sua completa remoção.

A eletromiografia realizada com eletrodos de superfície demonstrou ser um método prático para avaliar os movimentos faciais e pelo fato de permitir acompanhar a evolução do paciente de forma inócua e sem riscos ${ }^{26-32}$.

Um estudo demonstrou que a EMG superou a avaliação clínica para a determinação de sincinesias discretas que não foram vistas à inspeção visual ${ }^{33}$. No entanto, este estudo utilizou eletrodos de agulha para a avaliação eletromiográfica, na prática fonoaudiológica este tipo de eletrodo não é utilizado.

Acredita-se também que a eletromiografia de superfície será útil para detectar sinais de contratura e sincinesias em casos de sequelas de PFP, como referido pelos autores ${ }^{23}$, esta hipótese, entretanto está sendo motivo de nova pesquisa.

\section{CONCLUSÃO}

A eletromiografia de superfície mostrou diferença estatisticamente significante entre os valores eletromiográficos dos pacientes com paralisia facial periférica ( $\mathrm{HB} \mathrm{VI}$ ) e em indivíduos normais para todos os segmentos da face.

\begin{abstract}
Purpose: to study the surface electromyographic activity of frontal, orbicular occuli, orbicular oris and zigomatycs muscles in normal subjects and in peripheral facial paralysis patients. Methods: six volunteers with no facial paralysis history as well as six peripheral facial paralysis patients were evaluated with electromyography using superficial electrodes. Maximum effort muscle activity and symmetry index were measured for the voluntary movements such as: raising eyebrows, eyes closing, smiling, puckering lips. Results: it was found out that in normal subjects the muscle activity values were similar between the two sides of the face, showing that the facial nerve integrity is fundamental to the balance of facial mimics. In facial paralysis patients, the mean electromyographic values for both sides of the face were significantly different (evidencing the lack of facial nerve information to the muscles). Conclusion: the electromyographic results showed a statistically significant difference between the two sides of the face in the normal subjects and in facial paralysis patients.
\end{abstract}

KEYWORDS: Facial Paralysis; Electromyography; Facial Nerve

\section{REFERÊNCIAS}

1. Bento RF. Doenças do nervo facial. In: Bento RF, Miniti A, Marone SAM. Tratado de otologia. 1. ed. São Paulo: Edusp; 1998. p. 427-59.

2. Diels HJ. Facial paralysis: is there a role for a therapist? Facial Plast Surg. 2000; 16(4):361-4.

3. Goffi-Gomez MVS, Bogar P, Bento RF. Exercícios miofuncionais e paralisia facial periférica idiopática. Rev Bras Otorrinolaringol. 1996; 62(4):322-30.

4. Ferreira DF, Goffi-Gomez MVS. Perfil funcional na seqüela da paralisia facial periférica. I Congresso Otorrinolaringologia do Hospital das Clínicas da Faculdade de Medicina da Universidade de São Paulo; Junho; 1999.
5. House JW, Brackmann DE. Facial nerve grading system. Otolaryngol Head Neck Surg. 1985; 93(2):146-7.

6. Ross BG, Fradet G, Nedzelski JM. Development of a sensitive clinical facial grading system. Otolaryngol Head Neck Surg. 1996; 114(3):380-6.

7. Bernardes DFF, Goffi-Gomez MVS, Pirana S, Bento RF. Perfil funcional nos indivíduos com paralisia facial tratados em uma abordagem miofuncional. Pró-Fono. 2004; 16(2):151-8.

8. Sittel C, Stennert E. Prognostic value of electromyography in acute peripheral facial palsy. Otol Neurotol. 2001; 22(1):100-4.

9. Tomé MC, Marchiori SC. Análise eletromiográfica dos músculos orbiculares superior e inferior da boca 
em crianças respiradoras nasais e bucais durante a emissão de sílabas. Pró-Fono. 1999; 11(1):1-7. 10. Rodrigues KA. Eletromiografia nos masseteres no apertamento dental e na mastigação em indivíduos com e sem maloclusão. [dissertação] São Paulo (SP): Pontifícia Universidade Católica de São Paulo; 2001. 127p.

11. Rahal ACR. Eletromiografia dos músculos masseteres e supra-hióideos em mulheres com oclusão normal e com má-oclusão classe I de Angle durante a fase oral da deglutição. Rev CEFAC. 2004; 6(4):370-5.

12. Rahal A, Pierotti S. Eletromiografia e cefalometria na fonoaudiologia. In: Ferreira LP, Befi-Lopes DM, Limongi SCO, organizadores. Tratado de fonoaudiologia. São Paulo: Roca; 2004. p. 237-53.

13. Andrade CRF, Sassi FC, Ercolin B. Modelamento da fluência com o uso da eletromiografia de superfície: estudo piloto. Pró-Fono. 2008 abr-jun; 20(2):129-32.

14. Rahal A, Goffi-Gomez MVS. Avaliação eletromiográfica do músculo masseter em pessoas com paralisia facial periférica de longa duração. Rev. CEFAC. 2007; 9(2):207-12. dx.doi.org/ S1516-18462007000200009

15. Santini C. Distant learning for facial nerve retraining. Non-surgical rehabilitation. Anais do Simpósio do Nervo Facial. São Francisco. Session C; 2001.

16. Henkelmann TC, May M. Physical therapy and neuromuscular rehabilitation. In: May M, Schaitkin BM. The facial nerve. 2. ed. Thieme. 2000. p. 301-18.

17. Nakamura K, Toda N, Takeda N. Biofeedback rehabilitation for prevention of synkinesis after facial nerve palsy. Anais do Simpósio do Nervo Facial. São Francisco. Session C; 2001.

18. Bernardes DFF, Franco MZ. Restabelecendo a estética do sorriso usando o biofeedback eletromiográfico de superfície aliado a terapia miofuncional método $M Z$, estudo de caso. [monografia] São Paulo (SP): CEFAC - PósGraduação em Saúde e Educação; 2008.

19. Rosson GD, Redett RJ. Facial palsy: anatomy, etiology, grading, and surgical treatment. J Reconstr Microsurg. 2008 Aug; 24(6):379-89.

\section{DOI: 10.1590/S1516-18462009005000052 \\ RECEBIDO EM: 02/10/2008 \\ ACEITO EM: 06/05/2009}

Endereço para correspondência:

Daniele Fontes Ferreira Bernardes

Rua Caraíbas, 823

São Paulo - SP

CEP: 05020-000

E-mail: danifontes@bol.com.br
20. Calais LL, Gomez MVSG, Bento RF, Comerlatti LR. Avaliação funcional da mímica na paralisia facial central por acidente cerebrovascular. PróFono. 2005 maio-ago; 17(2):213-22.

21. Andrade CRF, Sassi FC, Juste FS, Meira MIM. Atividades de fala e não-fala em gagueira: estudo preliminar. Pró-Fono. 2008; 20(1):67-70.

22. Grisolía FM, Ferrary T. Development of an intraoral device for facial muscle retraining and its clinical application. Acta Odontol Latinoam. 2007; 20(1):49-54.

23. Goffi-Gomez MVS, Vasconcelos LGE, Bernardes DFF. Intervenção fonoaudiológica na paralisia facial. In: Ferreira LP, Befi-Lopes DM, Limongi SCO, organizadores. Tratado de fonoaudiologia. São Paulo: Roca; 2004. p.512-26.

24. Burres SA. Facial biomechanics: the standards of normal. Laryngoscope. 1985; 95(6):708-14.

25. Deleyiannis FW, Askari M, Schmidt KL, Henkelmann TC, Vanswearingen JM, Manders EK. Muscle activity in the partially paralyzed face after placement of a fascial sling: a preliminary report. Ann Plast Surg. 2005 Nov; 55(5):449-55.

26. Vanswearingen J. Facial rehabilitation: a neuromuscular reeducation, patient-centered approach. Facial Plast Surg. 2008 May; 24(2):250-9. 27. Novak CB. Rehabilitation strategies for facial nerve injuries. Semin Plast Surg. 2004; 18(1):47-51. 28. Castelo PM, Gavião MBD, Pereira LJ, Bonjardim LR. Avaliação ultra-sonográfica dos músculos mastigatórios e dimensões faciais em crianças com oclusão normal e mordida cruzada posterior unilateral. Rev. CEFAC. 2007; 9(1):61-71. dx.doi. org/S1516-18462007000100009

29. Vianna-Lara MS, Caria PHF. Electromyographic analysis of the upper lip in nose and mouth breathers. Braz J Oral Sci. 2006; 5(19):1203-8.

30. Cattoni DM, Fernandes FD, Di Francesco RC, Latorre MRDO. Características do sistema estomatognático de crianças respiradoras orais: enfoque antroposcópico. Pró-Fono. 2007 out-dez; 19(4):347-51.

31. Gomes CF, Trezza EMC, Murade ECM, Padovani CR. Surface electromyography of facial muscles during natural and artificial feeding of infants. J Pediatr. 2006; 82(2):103-9.

32. Nyqvist KH, Ewald U. Surface electromyography of facial muscles during natural and artificial feeding of infants: identification of differences between breast-, cup- and bottle-feeding. J Pediatr.2006; 82(2):85-6.

33. On AY, yaltirik hp, Kirazli Y. Agreement between clinical and electromyographic assessments during the course of peripheric facial paralysis. Clin Rehabil. 2007; 21(4):344-50. 